\title{
On the Jefimenko's Non-Einsteinian Clocks and Synchronicity of Moving Clocks
}

\author{
Andrew Chubykalo, Augusto Espinoza \\ Unidad Académica de Física, Universidad Autónoma de Zacatecas, Zacatecas, México \\ Email: achubykalo@yahoo.com.mx
}

How to cite this paper: Chubykalo, A. and Espinoza, A. (2017) On the Jefimenko's Non-Einsteinian Clocks and Synchronicity of Moving Clocks. Journal of Modern Physics, 8, 439-447.

https://doi.org/10.4236/jmp.2017.84028

Received: February 3, 2017

Accepted: March 6, 2017

Published: March 9, 2017

Copyright () 2017 by authors and Scientific Research Publishing Inc. This work is licensed under the Creative Commons Attribution International License (CC BY 4.0).

http://creativecommons.org/licenses/by/4.0/

\begin{abstract}
In this work we analyze the concept of time dilation in its application to the rate of moving clocks. The rates of two equiform elementary electromagnetic clocks of different orientations relative to their direction of motion are computed on the basis of relativistic transformations of force and coordinates for the case when the clocks are at rest in a stationary reference frame and for the case when they are moving at constant speed relative to the stationary reference frame. It is shown that, although both clocks run slower when they are moving than when they are at rest, the rate of the moving clocks is affected by their orientation relative to their direction of motion, rather than by the kinematic (relativistic) time dilation as it is now generally assumed. The implication of this result for the experimental proofs of the existence of the kinematic the dilation is discussed.
\end{abstract}

\section{Keywords}

Synchronicity, Time Dilation, Length Contraction

\section{Introduction}

Albert Einstein in his famous 1905 paper interpreted the Lorentz transformation equations for coordinates and time as indicating that time is kinematically "dilated" in systems that move with respect to the systems considered to be stationary [1]. As a physical entity, time is defined in terms of specific measurement procedures, which may be described simply as "observing the rate of clocks". It is now generally assumed that because of time dilation, the rate of all moving clocks is slower by the factor $\gamma=\left(1-v^{2} / c^{2}\right)^{1 / 2}$ than the rate of the same stationary clocks. However, a clock is a physical apparatus or device and is subject to the laws of physics in accordance with which the clock is constructed. Therefore, if a clock slows down when it moves, its slower rate should be explainable on the 
basis of the specific laws responsible for the operation of the clock and on the basis of relativistic transformations applicable to these laws.

In particular, if mechanical or electromagnetic forces are involved in the operation of a clock, then the rate of the moving clock should be explainable on the basis of these forces and on the basis of the relativistic force transformation equations (for the derivation of these equations see, for example, [2])

$$
\begin{gathered}
F_{x}^{\prime}=F_{x}-\frac{v u_{y}}{c^{2}\left(1-v u_{x} / c^{2}\right)} F_{y}-\frac{v u_{z}}{c^{2}\left(1-v u_{x} / c^{2}\right)} F_{z}, \\
F_{y}^{\prime}=\frac{1}{\gamma\left(1-v u_{x} / c^{2}\right)} F_{y}, \\
F_{z}^{\prime}=\frac{1}{\gamma\left(1-v u_{x} / c^{2}\right)} F_{z},
\end{gathered}
$$

where the primes indicate force components observed in a moving frame; the unprimed quantities are quantities observed in the stationary reference frame (laboratory); $v$ is the velocity of the moving reference frame relative to the laboratory; $u_{x}$ is the $x$-component of the velocity of the force-experiencing object relative to the laboratory; and $c$ is the velocity of light.

Furthermore, if the functioning of a clock depends on its geometrical parameters, then the rate of the moving clock may depend on the relativistic transformation equations for coordinates [2]

$$
\begin{gathered}
x^{\prime}=\gamma(x-v t), \\
y^{\prime}=y, \\
z^{\prime}=z .
\end{gathered}
$$

In this connection it is important to note that in Equations (1)-(6) the $x$ components transform differently from the $y$ and $z$ components. Therefore, if two synchronous stationary clocks, one oriented in the $x$ direction, the other oriented in the $y$ or $z$ direction, are placed in a moving reference frame, the clocks may become asynchronous in that reference frame, because both the forces acting on differently oriented clocks and the linear dimensions of differently oriented clocks will transform differently.

The purpose of this paper is to verify the above considerations regarding the rate of moving clocks by actual calculations and to discuss the implication of the obtained results for the experimental proof of kinematic time dilation. In the calculations that follow, we shall compute and compare the rates of two stationary elementary electromagnetic clocks with the rates of the same moving clocks. The operation of our clocks will be based on the interaction between a field-experiencing electric point charge and different field-producing electric point charge configurations.

\section{Calculations}

Clock \#1. Consider two positive point charges $q_{1}$ of the same magnitude located at the points $\pm a$ of the $z$-axis. Let the charges be fixed in the 
laboratory. A negative point charge $q_{2}$, whose mass is $m_{0}$, is placed at a point $y$ of the $y$-axis close to the origin (so that $y \ll a$ ) and is constrained to move only along the $y$-axis ${ }^{1}$ (Figure 1 ).

The electric field produced by the charges $q_{1}$ at the location of the charge $q_{2}$ is

$$
\boldsymbol{E}=\frac{q_{1} y}{2 \pi \varepsilon_{0}\left(a^{2}+y^{2}\right)^{3 / 2}} \boldsymbol{j}
$$

where $\varepsilon_{0}$ is the permittivity of space and $\boldsymbol{j}$ is a unit vector in the $y$ direction. Since $y \ll a$ (as we can see from Figure 1), we can neglect $y^{2}$ in the denominator of Equation (7). The force experienced by $q_{2}, \boldsymbol{F}=-q_{2} \boldsymbol{E}$, is then essentially

$$
F=-\frac{q_{1} q_{2} y}{2 \pi \varepsilon_{0} a^{3}} \boldsymbol{j} .
$$

This is a linear restoring force. Therefore our system of the three charges constitutes a simple harmonic oscillator, and the charge $q_{2}$ oscillates with the period

$$
T=2 \pi\left(\frac{m_{0}}{F / y}\right)^{1 / 2}=(2 \pi a)^{3 / 2}\left(\frac{m_{0} \varepsilon_{0}}{q_{1} q_{2}}\right)^{1 / 2} .
$$

Clearly, this system of the three charges may be considered to constitute a clock and can be used for measuring time in terms of the period of oscillations $T$.

Let us now assume that the three charges are placed in a reference frame moving along the $x$-axis with constant velocity $\boldsymbol{v}=v \boldsymbol{i}$ relative to the laboratory. The force $\boldsymbol{F}^{\prime} \square$ acting on $q_{2}$ as observed in the moving reference frame (where the charges $q_{1}$ are at rest now) is the same as that given by Equation (8), except that $y$ and $a$ are now written with primes, that is

$$
\boldsymbol{F}^{\prime}=-\frac{q_{1} q_{2} y^{\prime}}{2 \pi \varepsilon_{0} a^{\prime 3}} \boldsymbol{j}
$$

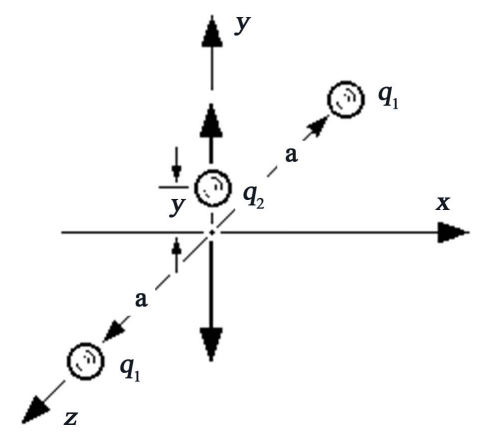

Figure 1. A negative point charge $q_{2}$ oscillates along the $y$-axis under the action of two fixed positive point charges $q_{1}$. This system constitutes an elementary electromagnetic clock.

${ }^{1}$ The charge must be constrained to stay on the axis because otherwise it is unstable with respect to a lateral displacement. 
To find the force acting on $q_{2}$ in the moving clock as observed from the laboratory, we use Equations (2), (5) and (6) combined with Equation (10). Let us assume that the velocity $v$ of the moving reference frame is much larger than the maximum oscillatory velocity of $q_{2}$. The velocity $\boldsymbol{u}$ of $q_{2}$ relative to the laboratory is then equal to $v$, and its $x$ component, $u_{x}$, is then simply $v$. Hence Equation (2) becomes

$$
F_{y}^{\prime}=\frac{1}{\gamma\left(1-v^{2} / c^{2}\right)} F_{y}=\gamma F_{y},
$$

from which we obtain

$$
F_{y}=\frac{F_{y}^{\prime}}{\gamma} .
$$

Substituting Equation (10) into Equation (12) and taking into account that, by Equations (5) and (6) $)^{2}, y^{\prime}=y$ and $a^{\prime}=a$, we obtain for the force acting on $q_{2}$ in the moving clock as observed from the laboratory

$$
\boldsymbol{F}=-\frac{q_{1} q_{2} y}{2 \pi \varepsilon_{0} a^{3}} \boldsymbol{j} .
$$

Taking into account that the mass of the moving charge $q_{2}$ (actually its transverse mass, See, for example, H. Goldstein [3]) is

$$
m_{\text {moving }}=\gamma m_{0},
$$

and using Equation (9) with $m_{0}$ in it replaced by $m_{\text {moving }}$, we obtain for the period of oscillations of the charge $q_{2}$ in our moving clock \#1 as observed from the laboratory

$$
\begin{aligned}
T_{\text {moving }} & =2 \pi\left(\frac{m_{\text {moving }}}{F / y}\right)^{1 / 2}=(2 \pi a)^{3 / 2}\left(\frac{\gamma^{2} m_{0} \varepsilon_{0}}{q_{1} q_{2}}\right)^{1 / 2} \\
& =\gamma(2 \pi a)^{3 / 2}\left(\frac{m_{0} \varepsilon_{0}}{q_{1} q_{2}}\right)^{1 / 2} .
\end{aligned}
$$

Comparing Equation (15) with Equation (9), we find that

$$
T_{\text {moving }}=\gamma T_{\text {stationary }},
$$

so that the rate of our moving clock \#1 is longer by the factor $\gamma$ than its rate when it is stationary.

Clock \#2. This clock is the same as clock \#1 except that the field-producing charges $q_{1}$ are now placed along the $x$-axis at the points $\pm a$ of the axis (Figure 2). The point charge $q_{2}$ is again on the $y$ axis close to the origin, so that $y \ll a$.

Clearly, when clock \#2 is at rest in the laboratory reference frame, the period of oscillations of $q_{2}$ is the same as that given by Equation (9) for clock \#1, that is

$$
T=2 \pi\left(\frac{m_{0}}{F / y}\right)^{1 / 2}=(2 \pi a)^{3 / 2}\left(\frac{m_{0} \varepsilon_{0}}{q_{1} q_{2}}\right)^{1 / 2} .
$$

${ }^{2}$ Observe that $a$, being the $z$ coordinate of $q_{1}$, transforms according to Equation (6). 


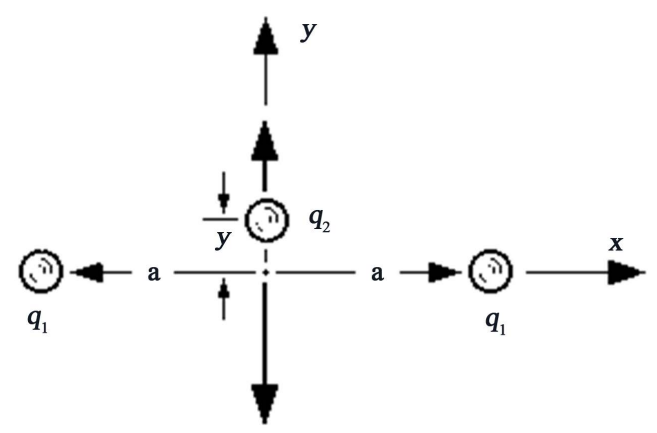

Figure 2. A negative point charge $q_{2}$ oscillates along the $y$ axis under the action of two fixed positive point charges $q_{1}$. This system constitutes an elementary electromagnetic clock.

Let us now assume that clock \#2 is placed in a reference frame moving along the $x$ axis with velocity $v=v i$ relative to the laboratory. As observed in that reference frame (where the charges $q_{1}$ are at rest now), the force $\boldsymbol{F}^{\prime}$ acting on $q_{2}$ is the same as that given by Equation (10), that is

$$
\boldsymbol{F}^{\prime}=-\frac{q_{1} q_{2} y^{\prime}}{2 \pi \varepsilon_{0} a^{\prime 3}} \boldsymbol{j}
$$

To find the force acting on the moving $q_{2}$ as observed from the laboratory, we use Equations (2), (5) and (4) combined with Equation (18). As before, we assume that the velocity $\boldsymbol{v}$ of the moving reference frame is much larger than the maximum oscillatory velocity of $q_{2}$. The velocity $\boldsymbol{u}$ of $q_{2}$ relative to the laboratory is then again equal to $\boldsymbol{V}$, and its $x$ component, $u_{x}$, is simply $v$. Hence Equation (18), just like Equation (10), becomes

$$
F_{y}^{\prime}=\frac{1}{\gamma\left(1-v^{2} / c^{2}\right)} F_{y}=\gamma F_{y},
$$

from which we again obtain

$$
F_{y}=\frac{F_{y}^{\prime}}{\gamma} .
$$

Substituting Equation (18) into Equation (20) we have

$$
\boldsymbol{F}=-\frac{q_{1} q_{2} y^{\prime}}{\gamma 2 \pi \varepsilon_{0} a^{\prime 3}} \boldsymbol{j}
$$

where we still need to transform $y^{\prime}$ into $y$ and $a^{\prime}$ into $a$. This we do with the help of Equations (5) and (4).

First we notice that, by Equation (5),

$$
y^{\prime}=y,
$$

To find the relation between $a^{\prime}$ and $a$, we proceed as follows. Let us designate the positions of the two $q_{1}$ charges observed from the laboratory as $x_{\text {right }}=a$ and $x_{\text {left }}=-a$, where $x_{\text {right }}$ refers to the charge located to the right of the origin and $x_{\text {left }}$ refers to the charge located to the left of the origin. The distance between the two charges observed from the laboratory is then 


$$
2 a=x_{\text {right }}-x_{\text {left }} .
$$

Let the time of observation in the laboratory be $t$ (arbitrary time of observation). Using Equation (4), we have for the positions of the two $q_{1}$ charges measured in the moving reference frame in terms of their positions observed from the laboratory

$$
x_{\text {right }}^{\prime}=\gamma x_{\text {right }}-\gamma v t
$$

and

$$
x_{\text {left }}^{\prime}=\gamma x_{\text {left }}-\gamma v t .
$$

The distance between the two charges measured in the moving reference frame is then, by Equations (24), (25) and (23),

$$
2 a^{\prime}=x_{\text {right }}^{\prime}-x_{\text {left }}^{\prime}=\gamma x_{\text {right }}-\gamma v t-\gamma x_{\text {left }}+\gamma v t=\gamma\left(x_{\text {right }}-x_{\text {left }}\right)=\gamma 2 a,
$$

and hence

$$
a^{\prime}=\gamma a \text {. }
$$

Substituting $y^{\prime}$ and $a^{\prime}$ in Equation (21), we therefore obtain for the force acting on the charge $q_{2}$ in the moving clock as observed from the laboratory

$$
\boldsymbol{F}=-\frac{q_{1} q_{2} y}{\gamma^{4} 2 \pi \varepsilon_{0} a^{3}} \boldsymbol{j} .
$$

Taking into account that the mass of the moving charge $q_{2}$ is

$$
m_{\text {moving }}=\gamma m_{0},
$$

and using Equation (17) with $m_{0}$ in it replaced by $m_{\text {moving }}$, we obtain for the period of oscillations of the charge $q_{2}$ in our moving clock \#2 as observed from the laboratory

$$
\begin{aligned}
T_{\text {moving }} & =2 \pi\left(\frac{m_{\text {moving }}}{F / y}\right)^{1 / 2}=(2 \pi a)^{3 / 2}\left(\frac{\gamma^{5} m_{0} \varepsilon_{0}}{q_{1} q_{2}}\right)^{1 / 2} \\
& =\gamma^{5 / 2}(2 \pi a)^{3 / 2}\left(\frac{m_{0} \varepsilon_{0}}{q_{1} q_{2}}\right)^{1 / 2} .
\end{aligned}
$$

Comparing Equation (30) with Equation (17), we find that for clock \#2

$$
T_{\text {moving }}=\gamma^{5 / 2} T_{\text {stationary }},
$$

so that, in contrast to Clock \#1, the rate of the moving Clock \#2 is dilated by the factor $\gamma^{5 / 2}$ rather than by the factor $\gamma$.

\section{Discussion}

The calculations just presented reveal two properties of the rate of moving clocks: 1) moving clocks run slower than the same stationary clocks; 2) differently constructed (or oriented) synchronous stationary clocks may run at different rates and therefore may become asynchronous when placed into a moving reference frame. Quantitatively, our first clock behaves in accordance with the idea of the kinematic (relativistic) time dilation (the moving clock runs $\gamma$ times slower than the stationary clock), but our second clock behaves in stark 
disagreement with the idea of the kinematic time dilation (the moving clock runs $\gamma^{5 / 2}$ times slower than the stationary clock). And yet, the rate of the second clock is in accord with the fundamental relativistic transformation equations used in our calculations. To understand the behavior of the second clock, let us consider the electromagnetic forces responsible for the functioning of the two clocks.

As is known, the electric field produced by a moving point charge concentrates itself about the plane perpendicular to the direction of motion and decreases along the line of motion (see, for example [4]). Therefore, as seen by a stationary observer, the electric force acting on the charge $q_{2}$ due to the electric field of the charges $q_{1}$ in the moving clock \#1 is different from the electric force acting on the charge $q_{2}$ due to the electric field of the charges $q_{1}$ in the moving clock \#2. Likewise, the magnetic force acting on the charge $q_{2}$ (due to the magnetic field created by the charges $q_{1}$ in the moving clocks) is different in the two moving clocks. Thus, whereas the charge $q_{2}$ in the stationary clocks $\# 1$ and \#2 is subjected to the same force, and the clocks are therefore synchronous, in the moving clock \#1 the charge $q_{2}$ is subjected to forces different from the forces acting upon it in the moving clock \#2, and therefore the two moving clocks are no longer synchronous (for a detailed discussion and calculations of the electromagnetic forces acting in the differently oriented and differently constructed clocks see [5]). For this kind of clocks we would like to denominate as Jefimenko's non-Einsteinian clocks) ${ }^{3}$.

It may at first appear that the principle of relativity is violated by the fact that synchronous stationary clocks may become asynchronous when moving. This is not so. According to the principle of relativity, it is impossible to tell whether a particular inertial reference frame is "actually" moving or is stationary. The fact that synchronous clocks may become asynchronous when placed in a "moving" reference frame does not tell us which of the two frames is really moving, because the effect is reciprocal. It is easy to see that if we started with synchronous clocks resting in the "moving" reference frame, then placed them into the "stationary" reference frame and used transformation equations expressing unprimed force components and coordinates in terms of primed quantities, we would find that the clocks placed in the "stationary" reference frame would not be synchronous when viewed from the "moving" reference frame. Thus placing synchronous clocks into a different reference frame and finding that the clocks become then asynchronous provide no information on whether or not one or the other of these reference frames is "actually" moving or is stationary, which is in complete agreement with the principle of relativity.

There is, however, an important implication result as far as the proofs of the existence of the kinematic (relativistic) time dilation are concerned. It is now generally believed that the existence of kinematic time dilation has been demonstrated by actual experiments [6] [7] [8]. As has been already pointed out

${ }^{3}$ In his book [5] Jefimenko adduces also other kinds of clocks that do not run in accordance with Einstein's theory, e.g., $\gamma^{3 / 2}$-clock. 
by Jefimenko [9], and as is clear from the calculations presented in this paper, the experiments that are interpreted as proofs of the reality of kinematic time dilation may have a simple alternative interpretation in terms of velocitydependent forces present in the systems under consideration. Of course, we do not know what forces are responsible for the decay of elementary particles and we know little about the forces responsible for the functioning of atomic clocks. But there can be no doubt that the decay of elementary particles, as well as the action of atomic clocks, is controlled by some kind of forces. In the light of the calculations presented in this paper and of similar calculations presented elsewhere [5] [9], it is much more natural and prudent to interpret the experiments allegedly proving the reality of kinematic time dilation as manifestations of the existence of velocity-dependent forces and interactions in the systems under consideration.

Moreover, it is now clear that to prove the reality of kinematic time dilation it is necessary not only to demonstrate that moving clocks run slower by the factor $\gamma$ than the same stationary clocks, but it is also necessary to prove that the rate of the moving clocks is not affected by their mechanism, structure, orientation or by any other specific physical or geometrical property of the clocks.

In conclusion, in connection with the above, we would like to adduce here (or propose to consider) a curious paradox:

Imagine that in the system $K^{\prime}$, which moves with a constant velocity $v$ relative to the stationary system $K$, are installed two clocks: " $\gamma T_{\text {stationary }}$ "-clock ( $\gamma$ -clock) Equation (16) and “ $\gamma^{5 / 2} T_{\text {stationary }}$ "-clock ( $\gamma^{5 / 2}$-clock) Equation (31). The clocks are installed on a straight line perpendicular to the straight line $K^{\prime} K$ along which the system $K^{\prime}$ moves in the direction of the stationary system $K$, the clocks are equidistant from the line $K^{\prime} K$ and let these clocks are synchronized in $K^{\prime}$ At some distance from the clocks in the system $K$ are installed some "gimmick" with two sensors installed just like the clocks in $K^{\prime}$ on a straight line perpendicular to the straight line $K^{\prime} K$. Let these two clocks simultaneously at some arbitrary moment of time (in the system $K^{\prime}$ send light pulses in the directions of the corresponding sensors of "gimmick". Let a cat is placed in this device. The sensors are constructed so that if they simultaneously (in the system $K)^{4}$ receive the light pulses, it triggered an automatic gear which kills the cat in the "gimmick". If the light pulses will not received by the sensors at the same moment of time of the system $K$, the gear will not work and the cat will stay alive.

It is obvious that $\gamma$-clock and $\gamma^{5 / 2}$-clock once synchronized in the system $K^{\prime}$ will remain synchronized in this system constantly. Thus, from the point of view of the system $K^{\prime}$ pulses emitted by $\gamma$-clock and $\gamma^{5 / 2}$-clock simultaneously at some arbitrary moment of time in the system $K^{\prime}$ to the sensors of the "gimmick" reach the respective sensors simultaneously! And thus the poor cat will be killed. However, from the viewpoint of the system $K \quad \gamma$-clock and $\gamma^{5 / 2}$-clock go with different rate. It means that the pulses are emitted at the dif-

${ }^{4}$ In this system time is measured by the " $T_{\text {stationary }}$ "-clock. 
ferent moments of time in the system $K$ and from different distances from the system $K$ and will not reach the sensors simultaneously! Thus, from the viewpoint of the system $K$ the cat will stay alive! Let us name this cat as "Jefimenko's cat" (by analogy with the famous Shroedinger's cat).

As for the "Jefimenko's cat" yet it is not a "point of view". According to our calculations, the non-synchronicity of clocks is a purely objective phenomenon. Its essence is that stationary systems and moving systems are different physical systems, and so the physical phenomena occur in them in different ways.

\section{References}

[1] Einstein, A. (1905) Annals of Physics, 17, 891-921. https://doi.org/10.1002/andp.19053221004

[2] French, A.P. (1968) Special Relativity. Norton, New York, 237.

[3] Goldstein, H. (1951) Classical Mechanics. Addison-Wesley, Cambridge, 205.

[4] Jackson, J.D. (1999) Classical Electrodynamics. 3rd Edition, Wiley, New York, 559561.

[5] Jefimenko, O.D. (2004) Electromagnetic Retardation and the Theory of Relativity. 2nd Edition, Electret Scientific, Star City, 237-244, 251-253.

[6] Rossi, B. and Hall, D.B. (1941) Physical Review, 59, 223. Fisch, D.H. and Smith, J.H. (1963) American Journal of Physics, 31, 342.

Bailey, J., Borer, K., Combley, F., Drumm, H., Krienen, F., Lange, F., Picasso, E., von Ruden, W., Farley, W.J.M., Field, J.H., Flegel, W. and Hattersley, P.M. (1977) Nature, 268, 301.

[7] Hafele, J.C. and Keating, R.E. (1972) Science, 177, 166, 168.

[8] Takabayashi, Y. (2001) High Precision Spectroscopy of Helium-Like Heavy Ions with Resonant Coherent Excitation. Doctor Thesis, Tokyo Metropolitan University, Tokyo.

[9] Jefimenko, O.D. (1998) Z. Naturforsch, 53a, 977. Jefimenko, O.D. (1998) American Journal of Physics, 63, 454. https://doi.org/10.1119/1.17911

\section{Scientific Research Publishing}

Submit or recommend next manuscript to SCIRP and we will provide best service for you:

Accepting pre-submission inquiries through Email, Facebook, LinkedIn, Twitter, etc. A wide selection of journals (inclusive of 9 subjects, more than 200 journals) Providing 24-hour high-quality service

User-friendly online submission system

Fair and swift peer-review system

Efficient typesetting and proofreading procedure

Display of the result of downloads and visits, as well as the number of cited articles Maximum dissemination of your research work

Submit your manuscript at: http://papersubmission.scirp.org/

Or contact jmp@scirp.org 